\title{
PLAYGROUND \\ DISEÑOS E INFRAESTRUCTURAS SEGURAS PARA NUESTROS NIÑOS Y NIÑAS...UNA URGENCIA EN COSTA RICA
}

\author{
María Morera-Castro \\ Maestría en Recreación, Universidad de Costa Rica \\ Costa Rica \\ mmore@una.cr
}

\section{RESUMEN}

Este artículo tiene como propósito dar a conocer los parámetros necesarios para el diseño e implementación de campos de juego seguros para la población. Ya que por medio de los campos de juego se suministran oportunidades únicas de desarrollo integral, especialmente en la edad infantil. En estos espacios públicos, los niños y las niñas tienen que aprender a resolver conflictos para seguir adelante con su juego, su diversión y su desarrollo. Este espacio lúdico se convierte en un lugar de aprendizaje que conlleva procesos de formación y grandes beneficios sociales, emocionales, físicos, cognoscitivos, intelectuales y espirituales. Sin embargo, estos beneficios se ven disminuidos por la falta de conciencia de las compañías constructoras que los diseñan en lugares no aptos, poniendo en riesgo a la población, también por el poco interés de las comunidades y la población adulta, en optimizar las condiciones para su uso. Por lo cual, es de suma importancia antes, durante y después de la construcción de un campo de juego tomar en cuenta: el diseño, la construcción, el material, los implementos, los componentes y los procedimientos que se deben de tener para que cumplan con los requerimientos necesarios de seguridad, y sin dejar de lado el objetivo de su creación, o sea un área diseñada, equipada, localizada y exclusiva para el juego y que facilite la formación integral de la población. Es urgente, por tanto, que en Costa Rica se establezcan legislaciones claras que garanticen el cumplimiento en cuanto a la construcción, diseño y utilización de los playgrounds, sin poner en riesgo la salud, previniendo cualquier tipo de accidente y garantizando los derechos inalienables de cada costarricense.

PALABRAS CLAVES: campos de juego (playgrounds), normativas, diseño, infraestructura, seguridad.

\section{PLAYGROUNDS: \\ SAFE DESIGNS AND INFRASTRUCTURES FOR OUR CHILDREN... AN URGENT MATTER IN COSTA RICA}

\begin{abstract}
The purpose of this article is to offer the design and infrastructure parameters necessary to have safe playgrounds since they represent a unique opportunity to foster an integral development, particularly in children. In these public places, children learn to resolve conflicts to continue playing, having fun, and developing. These recreational areas then become learning places that foster the formation process and provide great social, emotional, physical, cognitive, intellectual, and spiritual benefits. However, such benefits are diminished by the lack of interest of the communities and the adult population to optimize playground conditions and by unscrupulous developers, who design playgrounds in inappropriate places putting the population at risk. Therefore, the following must be taken seriously into consideration before, during, and after the construction of a playground: design, construction, materials, equipment, components and the procedures to meet the necessary safety requirements and the objective for which they were created, that being an area designed, equipped, and located exclusively for playing that facilitates the integral development of the population. Consequently, it is urgent for Costa Rica to enact clear regulations that guarantee the construction, design, and use of playgrounds that do not put the population's health at risk, prevent accidents, and guarantee the inalienable rights of each Costa Rican.
\end{abstract}

KEY WORDS: playgrounds, regulations, design, infrastructures, safety. 


\section{Introducción}

"Si usted quiere saber cómo es un niño o niña, obsérvelos como juegan. Si usted está interesado en lo que ellos van a convertirse en un futuro, guíe su juego (Gallahue \& Vannier, 1978, p.42)".

El juego es una parte esencial en la vida del infante, ocupa casi todo el tiempo desde el momento en que se levanta hasta cuando se acuesta. Este es una actividad que beneficia, de forma integral, el aprendizaje de conductas, desarrollo de destrezas y conceptos específicos (Stillwell, 1987).

La Declaración Universal de los Derechos Humanos, adoptada y proclamada el 10 de diciembre de 1948 por la Asamblea General de las Naciones Unidas (1948), especifica en el artículo 24 que "toda persona tiene derecho, al disfrute del tiempo libre...", sin importar su condición. Las reglas de las Naciones Unidas para la Protección de los Menores Privados de Libertad son concretas en su principio general. En su Artículo 47, esta normativa dispone que las personas menores de edad deban contar diariamente con el tiempo suficiente para realizar actividades de esparcimiento, practicar ejercicios físicos al aire libre o jugar. Si el clima lo permite, se proporcionará normalmente una educación recreativa y física. Para estas actividades, se contará con terrenos e instalaciones apropiadas y el equipo necesarios (Pavía, Lorente, Quinteros y Verbic, 2002).

La legislación costarricense también es clara con respecto a los derechos que poseen nuestros infantes, adolescentes y personas jóvenes, en cuanto a la cultura, recreación y deporte. La Ley de la Niñez y la Adolescencia No. 7739, en los artículos 73 y 75 señala que las personas menores de edad tendrán derecho a jugar y participar en actividades recreativas, deportivas y culturales, que les permitan ocupar provechosamente su tiempo libre y que contribuyan a su desarrollo integral. Además se cita al Ministerio de Cultura, Juventud y Deporte; a las corporaciones municipales, como entes responsables para establecer las políticas necesarias y ejecutar las acciones pertinentes para facilitar a las personas menores de edad, los espacios adecuados en niveles comunitarios y nacionales, que les permitan ejercer sus derechos recreativos y culturales. Al igual, la infraestructura deportiva y recreativa oficial estará a disposición de estos grupos en condiciones de plena igualdad, de acuerdo con las reglamentaciones que se emitan, para la práctica del deporte, etc. (UNICEF y Defensoría de los Habitantes, 1998).

La Ley General de la Persona Joven No. 8261, en el artículo 4 incisos e) y k), establece como derechos de esta población la recreación, por medio de actividades que promuevan el uso creativo del tiempo libre, para que disfrute de una vida sana, feliz, el derecho a convivir en un ambiente sano y participar en acciones que contribuyan a mejorar su calidad de vida (UNFPA y Consejo de la Persona Joven, 2002).

Los campos de juegos se convierten por tanto, en un suministro de oportunidades no solo para el cumplimiento de las leyes anteriormente mencionadas, sino también de actividades gratas y constructivas que pueden favorecer a los diferentes grupos etarios, especialmente entre los seis y los catorce años (Butler, 1966).

Los playgrounds, para algunos, son solamente un espacio físico, mientras que para otros es un lugar de recreación pública, de fácil acceso, en el cual se pueden experimentar vivencias placenteras y promover el aprendizaje en un espacio lúdico (Rico, 2002). Butler (1996) sugiere que los campos de juego se ubiquen inmediatos a un centro educativo, debido a que, facilitan el acceso a los grupos escolares durante y después de las horas de clases, etc., se asegura su máximo uso, con lo que se convierte en un espacio comunitario. 
Las miradas disociadoras de los planificadores y especialistas, siguen viendo el patio escolar de juegos, las plazas, campos y espacios verdes como un problema autónomo del sistema educativo y las diseñadoras urbanas, la actividad recreativa diaria como un problema del sistema de salud (Lorente, Pavía, Quinteros y Verbic, 2002). . En un estudio realizado en Turquía se determinó que el $83.3 \%$ de los campos de juego estaban a la par de una calle y el $60 \%$ no tenían ninguna barrera o cerca para proteger al infante. La separación por edades sólo se encontró en el $12 \%$ de estos campos; un $62.5 \%$ de los y las niñas sí se encontraban bajo supervisión y el $95.8 \%$ del equipo no es seguro en estos campos de juego (ACIC, Gulbayrak y Turaci, 2004), las cifras son claras.

En Costa Rica, según Francisco Mora Protti (citado por Villegas, 2005), director de Urbanismo, del Instituto Nacional de Vivienda y Urbanismo (INVU), cada urbanización debe tener un área destinada a parques y zonas verdes que va entre el 5\% y el $20 \%$ del territorio. Los urbanizadores deben dejar los parques construidos y equipados. Posteriormente, pasan a control de las municipalidades, las cuales deben darles mantenimiento. Además según el Reglamento para el Control Nacional de Fraccionamientos y Urbanizaciones, los campos de juego deben contar con un frente mínimo de 10 metros. No debe tener zonas de difícil vigilancia, ni debe ubicarse frente a una carretera principal, si está cerca de un río debe tener una malla de seguridad, y cuando colinda con viviendas una tapia, no se pueden ubicar a más de 300 metros de la casa más alejada, la topografía debe ser similar a la del resto de la urbanización y la mitad de su espacio será destinado a infantes (0 a 12 años), aspectos que con solo darse un paseo o visitar algunos de estos campos no se están cumpliendo. Las únicas normativas legales con que cuenta Costa Rica actualmente para regular estos espacios de juego es el Reglamento mencionado anteriormente y la Ley No. 4240 de Planificación Urbana (1978) en los artículo 24, 27 y 41.

Este artículo tiene como propósito dar a conocer las normativas necesarias para el diseño e implementación de campos de juegos seguros para la población costarricense, con el fin de prevenir los accidentes.

\section{Diseño e infraestructura de los campos de juegos}

El primer punto por tomar en cuenta para el diseño y construcción de los campos de juego es el objetivo y meta que persiguen: suministrar oportunidades en el desarrollo de las destrezas necesarias para moverse eficientemente, ayudar compartir y afianzar la visión de mundo de las personas (Booth, 1988).

Con base en lo anterior, es importante recordar que cada campo de juego presentará peculiaridades de acuerdo con el terreno, la topografía, los árboles o arbustos, arroyos, el tipo de barrio, las necesidades de la gente, los fondos disponibles para el fomento y mantenimiento y la naturaleza que los rodea, los cuales se deberán tomar en cuenta antes de planificarlos.

Una vez determinados estos aspectos, existen algunos factores importantes que se deben añadir al proceso, para que la eficacia, la imaginación y la apariencia tomen forma.

Estos son:

a) Uso eficaz de todo el terreno, por medio del establecimiento de la función y utilidad de cada superficie de este.

b) Espacio adecuado para las instalaciones, que sean un amplio, el cual garantice la seguridad y el juego satisfactorios.

c) Fácil supervisión u operación, pues los campos de juego usualmente la exigen.

d) Accesibilidad para las personas participantes. 
e) Utilización de los accidentes geográficos. Una ladera puede ser utilizada como zona de supervisión; un grupo de árboles como descanso y una superficie amplia, llana para el campo de juego.

f) Seguridad. Esta se puede lograr mediante la cuidadosa disposición de aparatos o juegos por la ubicación adecuada de caminos y senderos en relación con los principales rasgos geográficos.

g) Economía en la construcción: mediante un adecuado y cuidadoso planeamiento las operaciones costosas e incluso las más sencillas se pueden realizar a un bajo costo por medio de la utilización de todos los recursos disponibles.

h) Economía en el mantenimiento, un planeamiento cuidadoso significa cortar el césped, podar los arbustos, limpiar las piletas, estructuras y cuidar cada detalle de un campo de juego.

i) Comodidades para las personas que usan el campo. La comodidad y la conveniencia de la gente que utiliza los campos de juego, así como, las fuentes de agua potable, los lugares para sentarse y los espacios de estacionamiento son aspectos esenciales del servicio.

j) El aspecto del campo de juego debe ser agradable, tanto interna, como externamente (Butler, 1966).

Los procedimientos y operaciones son esenciales para el desarrollo adecuado de todo campo de juego. Por ende, antes de iniciar cualquier proyecto se debe trabajar en la elaboración de un plan general, que debe ser preparado y aprobado por las autoridades pertinentes. Para asegurarse los resultados óptimos de este se recomienda contar con un recreacionista y un arquitecto familiarizados o especializados en zonas recreativas. A su vez, el personal que se hará cargo del mantenimiento y funcionamiento del lugar debe ser partícipe de este proceso (Butler, 1966).

Algunos aspectos importantes por tomar en cuenta en el desarrollo de este plan son:

a) Nivelación y desagües. La nivelación es una operación cara por lo que los costos se deben reducir al mínimo, utilizando correctamente la topografía. Los desagües, por su parte, tienen como objetivo quitar el exceso de agua superficial y subterránea que de otro modo impediría el uso recreativo del terreno.

b) Alisamiento. Las diferentes formas de actividades dentro de un campo de juego, exigen la utilización de diversos tipos de superficies. Un terreno de arena y aserrín proporcionan un almohadillado para aquellas actividades donde se fomente el trepar. El césped es la mejor superficie para niñas y niños pequeños, la superficie de cemento, arcilla o material bituminoso son aptos para los campos que requieren de un rebote preciso y que estén sometidos a un uso intensivo. Las autoridades han experimentado mucho con varios materiales para superficies, pero aún no se ha encontrado una solución satisfactoria. Se han obtenido excelentes resultados con el uso del asfalto de corcho en el campo de juego infantil, sin embargo, este material es relativamente costoso.

c) Iluminación. Estas zonas no solo se iluminan para el juego en general, sino también para prolongar las horas de esparcimiento y aumentar el número de personas que las utilizan. A pesar de que el costo de los postes de acero y cables subterráneos es elevando, es conveniente emplearlos por apariencia y seguridad. No se debe olvidar que en la instalación del equipo adecuado, la altura y la ubicación cumplen una función esencial.

d) Senderos, caminos y estacionamiento. En terrenos pequeños los senderos ayudan a la gente a llegar cómoda y directamente al lugar deseado. En terrenos grandes pueden contribuir a formas de recreo como paseo, excursión o simplemente observación de lugares de especial belleza o naturales. Los estacionamientos deben estar diseñados para lograr un acceso más directo a los lugares que atraen o sirven a gran cantidad de gente. 
e) Cercas. Se recomienda que los campos de juego en los barrios permanezcan cerrados como medida de seguridad, funcionamiento eficaz y protección de las propiedades vecinas. Las cercas conviene que estén por lo menos a tres metros dentro de la línea de edificación para permitir el embellecimiento externo. Además se debe tener cuidado con la ubicación de portones, entradas y salidas.

f) Asientos. Estos contribuyen mucho a la comodidad de las personas que visitan el campo de juego o lugar recreativo, varían de tamaño y tipo dependiendo de la utilidad que se le vaya a dar. En el caso del campo de juego, estos se colocan para las personas que supervisen a los y las niños, por lo que se recomienda ubicarlos cerca de las zonas donde la población infantil se está recreando. Otros lugares donde son muy funcionales son en los baños, duchas, armarios y vestidores.

g) El agua. Destaca la belleza del paisaje, puede proporcionar refugio a las aves acuáticas y hace posible la utilización de baños, bebederos y sistemas de riego para el mantenimiento del lugar.

h) La belleza. Se ha considerado un aspecto esencial del parque vecinal, del grande y de la reserva, aunque este aspecto se ha descuidado en los campos de juegos y zonas deportivas. Sin importar cuán grande o pequeño sea el lugar, este embellecimiento se puede lograr mediante la prudente selección, ubicación y mantenimiento de plantas que se cultivan con facilidad en determinadas condiciones. Sin embargo, la belleza no solo se logra decorando el lugar sino que también la construcción y equipo deben ser proyectados, construidos y conservados de manera que contribuyan con la buena apariencia del campo.

i) Zona de vigilancia y de descanso. Para las personas adultas que acompañan o contribuyen con la supervisión del infante.

j) Otros equipos. Las mesas y los bancos pueden ser necesarios para los juegos tranquilos o para proporcionar lugares de picnic. En el campo de juego también se pueden contemplar plataformas para bailes o aeróbicos, un soporte para bicicletas, tableros con anuncios, cestos de basura, cajas para guardar suministros y equipos, entre otros (Butler, 1966; Hudson, Mack \& Thompson, 2000).

Al finalizar la planificación se debe revisar que las estructuras propuestas sean acordes con la edad de los menores y el grado de maduración, con el fin de que al practicar cualquier tipo de juego ya sea físico, social, creativo, cognitivo o tranquilo, se asegure el desarrollo motriz acorde con la población (López y Estapé, 2002).

\section{Equipamiento de un Campo de Juego}

A mediados de los años sesentas, trabajadores sociales, psicólogos, escultores y arquitectos trataron de atender los problemas relacionados con las instalaciones de juego para el público. El arquitecto neoyorquino, M.Paul Friedberg, emergería como el comandante de esta batalla. Su propuesta inicial, que ha tomado validez a través del tiempo, se fundamentó en un sistema de módulos enlazados, utilizando accesorios creativos plegados a una serie de opciones interactivas para que los niños y niñas lo usasen. La infraestructura propuesta por Frieldberg se ha convertido en la estructura estándar de estos tiempos porque él tomó en cuenta el parecer de la población infantil como participantes en el proceso de diseño (Booth, 1988).

Como bien lo realizó Paul Frieldberg, el equipo por utilizar debe ser diseñado y construido teniendo todo el tiempo la mentalidad de un niño, incluso explorando la estructura como si el lo fuese. La idea principal de esto es presentar al infante la mayor cantidad de caminos para subir, bajar, meterse, salirse a través de la estructura (Booth, 1988). Para determinar cuál pieza en especial es apropiada, se deben considerar los siguientes puntos: 
a) Debe proporcionar experiencias de movimiento que no son posibles sin el equipo.

b) Proveer los conceptos de la estructura del movimiento humano.

c) Motivar al infante a ser activo.

d) Proporcionar un grado de seguridad responsable (Stillwell, 1987).

Burton (1977) señala una guía para la selección de equipos. Ella sugiere que un equipo adecuado debe:

a) Estar acorde con la edad y estatura del niño o niña.

b) Crear un estímulo apropiado a los movimientos por edad.

c) Ser apropiados en los diferentes niveles de destrezas e intereses.

d) Ser duraderos, atractivos, multicolores y de fácil mantenimiento.

e) Permitir al infante el jugar de forma segura.

f) Satisfacer cada necesidad del menor en cuanto a explorar, jugar imaginariamente, moverse con energía, incrementar la condición y poder jugar tanto solo como con otros.

Larraz (citado por López y otros, 2002) indica que el campo de juego debe al menos ofrecer cuatro posibilidades: el jugar, mediante un soporte de materiales previamente diseñados, en un entorno seguro; posibilitar la participación cooperación y hacer jugar interviniendo con nuevas propuestas ya sea dadas por el niño (a) o por el adulto de acuerdo con la observación.

\section{Los Componentes del Campo de Juego}

Para la elaboración de los componentes del campo de juego contemporáneo, la persona manufacturera utiliza diferentes materiales, incluyendo ladrillo, piedra, plástico, concreto, acero y madera. La estructura base de madera se ha convertido en la elección más popular (Booth, 1988), a pesar de que tanto estos como el metal tienden a deteriorase con el tiempo. El plástico aparentemente logra mejor mantenimiento (Hudson, Thompson y Olse, 2004). Aunque en este campo se puede utilizar cualquier cosa para elaborar lo que se quiera, cajas de madera vacías, maderas de diversas longitudes, neumáticos de coche viejos, cajas de cartón, muebles usados, rollos de papel para paredes, trozos de cuerda, pintura entre otros, pueden considerarse componentes que facilitan la construcción del campo de juego, siempre y cuando cumpla con las normativas de seguridad y mantenimiento adecuados (Bengtsson, 1970).

Se recomienda que los campos de juego se construyan en formas irregulares, curvilíneas y alargadas y no estructuras cuadradas y lineales que fomentan la monotonía. En estas áreas, además, se pueden incorporar montículos y desniveles que fomenten la fantasía y la imaginación (López y otros, 2002).

Las superficies son de gran importancia. De arena blanda y con hierba resultan agradables para caminar y desarrollar el sentido del tacto. Las de concreto, a pesar de ser duras y lisas, son útiles para los juegos que implican bicicletas, carretillos para empujar y coches de juguete. Se recomienda a su vez que sea ondulado, ya que resulta mucho más interesante que uno a nivel. Los troncos de madera de diversas formas y tamaños son gratos complementos, dado que ellos dan vida a una superficie (Bengtsson, 1970).

Otro aspecto que dará interés y vida a un campo de juego es el material suelto, que va a satisfacer las exigencias creativas e inventivas del propio mundo de juego para la población infantil. Es esencial, también, crear una atmósfera de habitación o cierta intimidad en la mayoría de estos campos, debido a que la fantasía del niño parece brotar mejor cuando el mundo adulto está completamente excluido. Estas cercas internas pueden ser de muchas clases: terraplenes, plantas, 
tablazones, ladrillos y no requieren grandes alturas, ya que será vista desde los ojos de un niño (a) sentado (a) (Bengtsson, 1970).

Montar una plataforma en lo alto de un árbol, juegos de escalar, juegos de construir una cueva subterránea o tan siquiera brindar al infante las herramientas o materiales necesarios para que incursionen en el juego de aventura, sería otra opción por experimentar (López y otros, 2002).

Los toboganes actualmente han cambiado su diseño, son más anchos y de fondo fuerte, se aumentó la altura de los rieles laterales y se disminuyó el ángulo de inclinación. Los más seguros se fabrican con una curva al final y una salida paralela al suelo, esto hace más lento el ritmo de descenso y les permite a los y las niñas pisar el terreno con sus propios pies. Lastimosamente, aún existen algunos con un grado de inclinación superior a los cuarenta y cinco grados (Booth, 1988).

En cuanto a los pasamanos, también son más anchos, por lo cual permiten el tránsito en ambas direcciones. Algunos fabricantes han incluido zonas de descanso o rieles de pies durante el recorrido para los principiantes (Booth, 1988).

También se pueden diseñar áreas de juegos específicos, acordes con las necesidades de la población infantil desde los 5 a los 15 años:

1. Una zona de juego libre, grande, para realizar juegos tranquilos o bien andar en bicicleta, patinar, entre otros para niños (as) de 5 a 10 años.

2. Una zona de juegos equipada con columpios, toboganes, escaleras, cuerdas entre otros, para niños y niñas de 5 a 10 años.

3. Una zona de juegos de pelota para niños (as) de 10 a 16 años, se recomienda rodearlas de vallas para mayor seguridad.

4. Una o varias zonas grandes de juego cubierto para adolescentes de 12 a 16 años (Bengtsson, 1970).

Según la Ley No. 7600 de Igualdad de Oportunidades de Costa Rica, en el artículo 41 y 54, dice que se deben construir, ampliar o remodelar los edificios, parques, aceras, jardines, plazas, vías, servicios sanitarios y otros espacios de propiedad públicos para que cumplan con las reglamentaciones establecidas. Asimismo, garantizar que dichos espacios físicos donde se realicen actividades culturales, deportivas o recreativas sea de accesibilidad a todas las poblaciones. Por cuanto, los campos de juego no son excluyentes a esta ley y también deben eliminar las barreras arquitectónicas en la accesibilidad mediante rampas de anchura, pendiente y pavimentos aconsejables para las sillas de ruedas, agarraderas y demás modificaciones que sean necesarias para cumplir con el principio de igualdad de oportunidades y accesibilidad a los servicios (Lépiz y Jiménez, 2000).

\section{Normas de Seguridad para la prevención de accidentes en los campos de juego}

Según O’Brien (1998) las mayores causas de accidentes en los campos de juego son el uso inapropiado del equipo, la falta de supervisión de mantenimiento, el cual representa el 77\%. Por su parte, la Consumer Product Safety Commission (CPSC) (citado por Hudson y otros, 2004) indica que la población infantil es herida en gran número, en estos espacios públicos al escalar, columpiarse o deslizarse en los toboganes. En otro estudio sobre los accidentes en los campos de juego realizado por Lillis y Jaffe (1997) se señala que, la atención más frecuente en los centros hospitalarios como resultado de estos accidentes son las fracturas en extremidades superiores; dos tercios de las hospitalizaciones son causadas por accidentes al escalar aparatos; los (as) niños (as) 
mayores se accidentan más escalando y los (as) menores en toboganes. La población infantil menor sufre de mayor cantidad de heridas en la cabeza.

A pesar de que los estudios anteriores establecen una serie de accidentes en los campos de juego un estudio de Petrido, Sibert, Dedoukou, Skalkidis y Trichopoulos (2002) señala que cerca del 50\% pueden prevenirse con un cambio en la estructura y el equipamiento. Esta reducción significativa puede lograrse también, con medidas tan simples como una supervisión más cercana e incentivando al infante a usar zapatos apropiados y utilizar equipo de protección cuando sea necesario.

Históricamente, las plazas y las zonas verdes han cumplido una función social y dentro de ellas el juego y la recreación. Actualmente la situación de inseguridad que se vive, ha hecho que las personas menores de edad queden atrapadas junto a sus familias en sus hogares, limitando así el uso de los campos de juego (Lorente y otros, 2002). Por tanto, la seguridad cumple un papel de mucha importancia. Se recomienda cerrar perimetralmente los campos de juego al menos a un metro de altura, con un reducido número de accesos para facilitar la supervisión o vigilancia desde afuera a las personas que supervisan. Estos cierres pretenden proteger al infante de las incursiones que puedan venir del exterior e impedir a su vez que se alejen (López y otros, 2002).

La construcción de los campos de juego no se deben realizar a lo largo de calles muy transitadas, ferrocarriles u otras barreras naturales o artificiales, debido a que estos crearían un factor de inseguridad a la hora del ingreso (Butler, 1966).

En caso de que el material sea de madera, se recomienda que esté sin aristas ni astillas. Si es de material sintético se debe verificar que los ángulos no sean peligrosos ni sobresalgan. No se recomienda el uso de hierro ya que se oxida fácilmente (López y otros, 2002).

Si la superficie únicamente es de cemento se sugiere colocar un piso sintético (caucho, poliuretano) o remplazarlo con tierra o areneros en zonas determinadas. Si se mantienen las zonas de arenas, esta debe ser removida y renovada periódicamente para que no se compacte y pueda provocar accidentes. Además se debe controlar la distancia entre aparatos y sus superficies mínimas de uso (Booth, 1988).

El campo de juego no debe poseer barreras arquitectónicas que limiten la accesibilidad, por lo que las rampas anchas, pendiente y pavimento facilitarán el ingreso de cualquier persona con discapacidad (López y otros, 2002).

La supervisión es otra norma de seguridad muy importante, esta se debe desarrollar sobre todo por medio de señales, letreros y haciendo conciencia de la importancia de la supervisión o de otras conductas que se quieran rescatar o sancionar en los campos de juego (Hudson y otros, 2004).

Actualmente los nuevos campos de juego se diseñan para edades entre los dos y los doce años, por lo que se establecen dos áreas: una para los dos y cinco años, otra entre cinco y doce años. Las áreas en donde se integren niños y niñas de dos a doce años, no son recomendadas (Hudson y otros, 2004).

Finalmente, Hudson y otros (2004) establecen que el mantenimiento será el éxito para proveer un lugar hermoso, seguro y agradable. Este es un factor indispensable y debe ser realizado por especialistas bajo la supervisión de entes encargados para tales efectos como es el caso de la "National Program for Playground Safety". 


\section{Conclusiones}

En las páginas anteriores se ha tratado de expresar el valor personal y social que poseen los campos de juego, como un requisito social y un atributo espiritual en la formación de los (as) menores de edad. Estos representan lugares de encuentro, de esparcimiento y distracción, de aprendizaje y catarsis para toda persona que directa o indirectamente gozan de su utilización.

Es importante recordar que antes de crear un nuevo campo de juego, se debe realizar un plan de acción que contemple todos los aspectos necesarios para la construcción, equipamiento, mantenimiento y control. A su vez se deben conocer las necesidades y características de la población para la cual son diseñados y construidos, ya que estos deben colaborar en la formación integral del individuo y no convertirse en un medio inseguro para su aprendizaje.

El cumplimiento de las normas de seguridad, la educación en el uso y el mantenimiento adecuado tanto de usuarios y usuarias como de las personas que administran o están a cargo, son esenciales para el buen funcionamiento y la prevención de accidentes en los campos de juego.

En Costa Rica, no se incluyen normativas detalladas, respecto a los espacios y tiempos destinados al ejercicio del derecho al juego. Es urgente, por tanto, que se establezcan legislaciones claras que garanticen su cumplimiento en cuanto a la construcción, diseño y utilización de los campos de juego, con el fin de contribuir en la formación integral de nuestra población, colaborar en la prevención de accidentes que ponen en riesgo la salud de nuestros futuros ciudadanos y ciudadanas; garantizar los derechos inalienables de los costarricenses relacionados con la recreación.

\section{Referencias Bibliográficas}

ACIC, Y., Gulbayrak, C., \& Turaci, G. (2004, febrero). Investigation of the level of safety and appropriateness of playgrounds in Elazing city in Turkey. International Journal Environ Health Resistence, 14(1), 75-82. Obtenido el 10 de febrero del 2005, de: http://www.ncbi.nlm.nih.gov/entrez/query.fcgi?cmd=pubmed\&dopt=Abstract\&list_uids=14 660120

Bengtsson, A. (1970). Parques y campos de juego para niños. Barcelona: LABOR.

Booth, N. (1988, October). Playgrounds: Safety and fun by design. Parks \& Recreation. 29-32.

Burton, E. (1977). The new physical education for elementary school children. Boston: Houghton Mifflin.

Butler, G. (1966). Principios y métodos de recreación para la comunidad. Volumen 1. Argentina: Bibliográfica OMEGA.

Declaración Universal de los Derechos Humanos. (1948, 10 de diciembre). Obtenido el 17 de mayo del 2005 de, http://www.unhchr/udhr/lang/spn.htm.

Fondo de Población de las Naciones Unidas (UNFPA) y el Consejo de la Persona Joven. (2002). Ley No. 8261 General de la Persona Joven. Costa Rica: Lara Segura \& Asoc.

Hudson, S. D., Thompon, D., \& Olsen, H. (2004). How safe are our playgrounds? Park \& Recreation, 39(4), 53-59. 
Hudson, S. D., Mack, M., \& Thompson, D. (2000). Play is safe. Steps to keeping playground surfacing safe for our children. Parks and Recreation, 35(4), 78-85.

Lépiz, O. y Jiménez S. (2000). Una Oportunidad para la Igualdad: Comentario a la Ley 7600 sobre la Igualdad de Oportunidades para las personas con discapacidad. Costa Rica: Gossestra Intl. S.A.

Ley No.4240 Planificación Urbana. (1978, 15 de noviembre). Obtenido el 14 de mayo del 2005, de: http://asamblea.racsa.co.cr/ley/leyes_nombre.htm

Lillis, K., \& Jaffe, D. (1997, abril). Playground injuries in children. Pediatric Emergency Care, 13(2), 149-153. Obtenido el 12 de febrero del 2005, de: http://www.ncbi.nlm.nih.gov/entrez/query.fcgi?cmd=Retrieve\&db+pudmed\&dopt=Abstract \&list_uids=9127428

López, M. y Estapé, E. (2002, Octubre). La zona de juegos, un espacio para la educación física en los centros escolares. Revista Digital Efdeportes Buenos Aires, 53(8). Obtenido el 3 de febrero del 2005, de: http://efdeportes.com/.

Lorente, M., Pavía, V., Quinteros, N. y Verbic, F. (2002, agosto). El derecho al juego. Revista DigitalEfdeportes Buenos Aires, 51(8). Obtenido el 3 de febrero del 2005, de: http://efdeportes.com/efd51/juego.htm

Morera, M., León, A. y Camacho J. (2005). Análisis de la legislación de Asociaciones, Fundaciones, Parques Nacionales, Recreativos y Campos de Juego en Costa Rica. Curso PF-4905 Recreación y su Legislación, Maestría en Recreación, Universidad de Costa Rica.

O’Brien, E. (1998). Playground safety: It is everybody is responsability. Parks and Recreation. 33 (4), 2.

Pavia, V. (1997, junio). El proyecto sobre las formas cotidianas de juego infantil. Revista Digital Efdeportes, Educación Física y Deportes, 5(2). Obtenido el 10 de febrero del 2005., De: http://www.efdeportes.com/efd5/vp5.htm

Petrido, E., Silbert, J., Dedoukou, X., Skalkidis, I., \& Trichopoulos, D. (2002). Injuries in public and private playgrounds: the relative contribution of structural, equipment and human factors. Revista Digital PudMed, 91(6), 691-697. https://doi.org/10.1111/j.16512227.2002.tb03304.x

Rico, C. (2002, 22-25 junio). Ponencia presentada en el Panel "El espacio público como generador de usos alternativos para la convivencia ciudadana en torno a la Recreación" dentro del III Foro Internacional de Parques realizado entre 22 y 25 de junio 2002, Bogotá. Obtenido el 3 de febrero del 2005, de: http://www.redcreación.org/articulos/espaciopublico_espacioludico.html

Stillwell, J. (1981). Leisure and Recreation Concepts, a Critical Analysis. Boston: Ally and Bacon, Inc.

Stillwell, J. (1987). Making and Using Creative Play Equipment. USA: Human Kinestics Publishers. 
Villegas, J. (2005, 30 enero). Áreas de recreo están en zonas de riesgo. La nación, pp. 5A.

UNICEF y Defensoría de los Habitantes. (1998). Código de la Niñez y la Adolescencia. Costa Rica: UNICEF. http://www.outdoorfunstore.com/playground-history.asp.

Fecha de recepción del artículo: 05 de marzo del 2007.

Fecha de aceptación del artículo: 17 de abril del 2007.

Fecha de publicación del artículo: 16 de julio del 2007.

\section{(c) (i) (9)}

Artículo de la Revista MHSalud de la Universidad Nacional, Costa Rica protegido por Licencia Creative Commons Attibution-NonComercial-NoDerivs 3.0 Costa Rica,

Para más información visite www.una.ac.cr/MHSalud

Permissions beyond the scope of this license may be available at revistamhsalud@una.cr 